\title{
2.5 Å Resolution Cryo-EM Structure of Human Apo-ferritin Using an Optimized Workflow for Volta Phase Plate
}

\author{
Kunpeng $\mathrm{Li}^{1}$, Thomas Klose ${ }^{1}$, Chen $\mathrm{Sun}^{1}$, Yue Liu ${ }^{1}$ and Wen Jiang ${ }^{1}$ \\ 1. Department of Biological Sciences, Markey Center for Structural Biology, Purdue University, West \\ Lafayette, IN.
}

The Volta phase plate (VPP) is an apparatus mounted in the back focal plane (BFP) of the objective lens that can significantly improve image contrast by changing the phase of unscatter central beam[1]. Phase shift induced by the VPP gradually increases with cumulative electron dose during image acquisition. In practice, the data acquisition workflow uses a small defocus instead of in-focus condition, changes the VPP spots after the phase shift has reached $\sim 0.8 \pi$ to avoid image blur[2]. Several test samples have been solved to near-atomic resolution, such as the proteasome $(\sim 700 \mathrm{kDa})$ at $3.2 \AA$ with in-focus imaging[3], and $2.4 \AA$ with defocused images using a Titan Krios equipped with a VPP and energy filter[2]. Additionally, the structure of human heavy chain apo-ferritin $(\sim 500 \mathrm{kDa})$ was solved to $3.0 \AA$ using a Titan Krios, VPP, and Cs-corrector with an overfocus workflow[4]. So far, the $3.2 \AA$ structure of haemoglobin, $64 \mathrm{kDa}$, is the smallest protein reconstructed with cryo-EM to date using the VPP[5]. This achievement brought single-particle cryo-EM closer to the predicted small mass limit[6].

Although the VPP has been commercially available in recent years, utilization of VPP for high resolution 3-D reconstruction has not been generally optimized, only a few research groups having published sub-3 $\AA$ structures with VPP. Here, we report our experience optimizing VPP usage to obtain sub-3 $\AA$ structures, including a $2.5 \AA$ structure of apo-ferritin. Currently, there is no consensus method in evaluating VPP quality. Thus, we used the Thon rings of the 2D or 1D power spectra of the amorphous carbon film as a VPP quality indicator. Tracking the change in the Thon rings resolution limit insert and extract the VPP provides a quantitative quality measurement for the VPP alignment. The VPP spots giving poor Thon rings are marked as bad spots and would not be used for data collection. We found that many spots close to the edge of the VPP slot have bad quality or cause large defocus errors in automated data collection with Leginon. By updating the spots layout from $5 \times 24$ to $4 \times 19$ spots, with sparser positions and further from the edge of the slot, the automated data collection works more robust. Additionally, it is critical to periodically check, and realign the new VPP spots according to the carbon surface profile of the VPP. Human light-chain apo-ferritin $(\sim 450 \mathrm{kDa})$ was used to test the performance of our newly installed VPP mounted on Titan Krios TEM equipped with a Gatan K2 direct electron detector. Details about data collection and processing are listed in Table 1. The structural analyses of apo-ferritin at $2.5 \AA ̊$ resolution are presented in Figure 1 .

The VPP can significantly enhance image contrast while preserving high resolution singal, making it a valuable tool for resolving sub-3 $\AA$ cryo-EM structures of small particles, $<500 \mathrm{kDa}$. In this work, we have spent significant effort optimizing the usage of the VPP for high resolution, automated data collection. The successful validation of this new workflow will add to the collective knowledge of VPP usage in high resolution cryo-EM.

References:

[1] R. Danev et al, PNAS 111 (2014), p. 15635. 
[2] R. Danev, D. Tegunov and W. Baumeister, eLife 6 (2017), e23006.

[3] R. Danev and W. Baumeister, eLife 5 (2016), e13046.

[4] X. Fan et al, Structure 25 (2017), p. 1623.

[5] M. Khoshouei et al, Nature Communications 8 (2017), p. 16099.

[6] R. Henderson, Quarterly Reviews of Biophysics 28(2) (1995), p. 171.

[7] S. Chen et al, Ultramicroscopy 135 (2013), p. 24.

Table 1. Cryo-EM data collection and data processing statistics

Sample Human light chain apo-ferritin: 50ug/ml, Buffer: 0.1M HEPES pH7.0

Grid $\quad$ R 0.6/1 300mesh UltraAufoil coated with graphene oxide film and chitosan

Plunger Gatan CP3, Relative humidity: $80 \%$, Blotting time: $6 \mathrm{~s}$

TEM Titan Krios, Gatan K2 direct electron detector, Volta phase plate

Phase plate Slot: 2, Spots: 29 37, Spots changing: 50 movies, C2/C3: 38.21/51.27

Imaging conditions 300kV, Mag: 29K, Dose: 7.4 e-/pixel/s, $0.511 \AA \AA$ pixel at super resolution mode, Defocus: -500nm, 10 frames/s, Exposure time: $5 \mathrm{~s}$

Data processing Movies_total: 440, Particles_clean: 191K, Particles_refine: 132K, Frames alignment: MotionCor2, CT̄F estimation: Gctf, Particle picking/2D/3D classification: Relion and CisTEM, Refinement: JSPR, Local resolution: resMap, Model fitting: Chimera, Model refinement: Coot and Phenix.real_space_refine

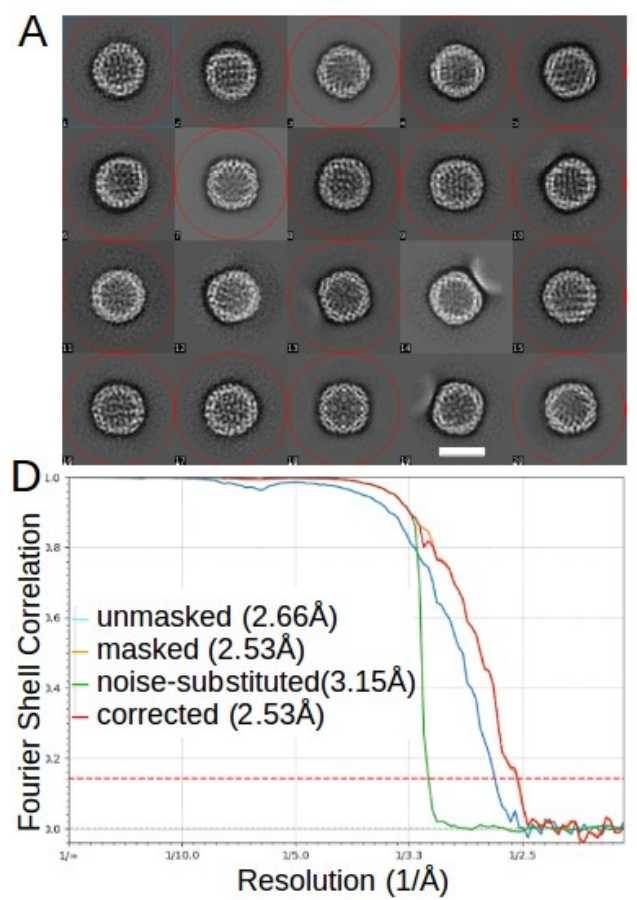

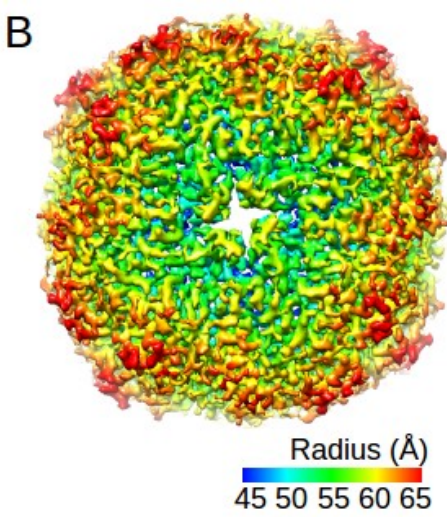

$E$
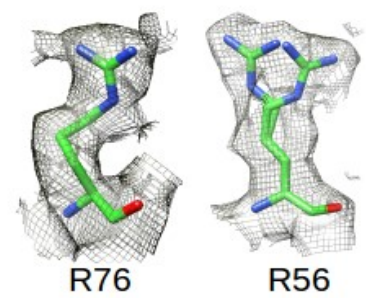
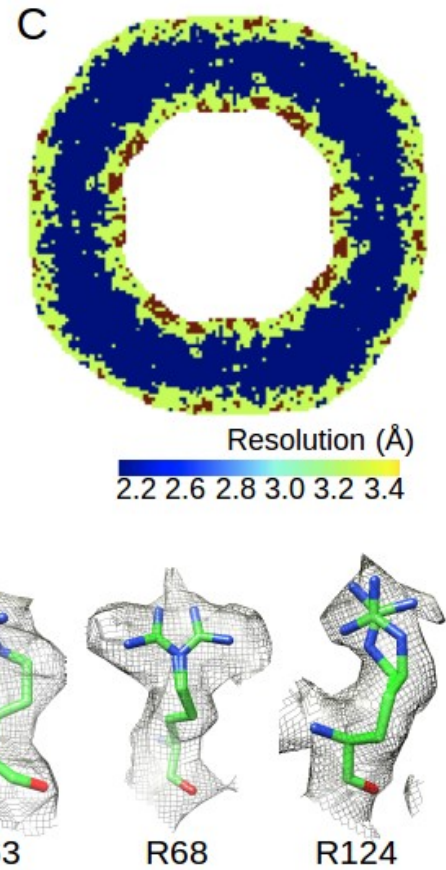

Figure 1. 3D reconstruction of VPP dataset of human light-chain apo-ferritin. (A) 2D class averages. Classes in red circle are selected for further processing. Scale bar: $10 \mathrm{~nm}$.(B) Radially colored surface view of the 3D map at $2.5 \AA$ resolution. (C) The center slice of the local resolution distribution of the map. (D) The true FSC curves of the 3D map[7]. (E) Representative side chains of Arginine, some of them with alternative conformation. 